\title{
Staphylococcus aureus: an underestimated factor in the pathogenesis of atopic dermatitis?
}

\author{
Leszek Blicharz, Lidia Rudnicka, Zbigniew Samochocki \\ Department of Dermatology, Medical University of Warsaw, Warsaw, Poland
}

Adv Dermatol Allergol 2019; XXXVI (1): 11-17

DOI: https://doi.org/10.5114/ada.2019.82821

\begin{abstract}
Atopic dermatitis is a common, recurrent pruritic dermatosis with a complex pathogenesis. It has been associated with disordered patterns of immunological response and impaired epithelial barrier integrity. These features predispose the patients to robust colonization of skin lesions by Staphylococcus aureus. Virulence factors of $S$. aureus (e.g. superantigens, $\alpha$ - and $\delta$-toxin, protein A) have been shown to exacerbate and perpetuate the course of atopic dermatitis. Novel therapeutic options with potential for restoring natural microbiome composition are being elaborated and may enter clinical practice in the future.
\end{abstract}

Key words: atopic dermatitis, pathogenesis, Staphylococcus aureus.

\section{Introduction}

The pathogenesis of atopic dermatitis (AD) is complex and remains unclear. It develops in subjects with a genetic predisposition under the influence of different environmental factors $[1,2]$. The skin microbiome and its fluctuations are directly associated with this disease. This review mainly focuses on the role of Staphylococcus aureus in the development and perpetuation of $A D$ (Figure 1).

\section{Microbiome - host interactions}

Numerous species of bacteria, viruses and fungi colonize human skin and are collectively defined as its microbiome [3, 4]. To understand the role of the skin microflora in the pathogenesis of $A D$, it is necessary to explore its qualitative and quantitative composition. The advent of Next Generation Sequencing (NGS) has facilitated the study on human microbiota. Grice et al. described the bacterial profile of 20 areas of the human skin thanks to the analysis of $16 \mathrm{~S}$ ribosomal RNA (16S rRNA) [5]. A total of 19 phyla were distinguished, four of which were dominant: Actinobacteria (59\% of total bacterial species, genera of this phylum - Micrococcus, Propionibacterium, Corynebacteria), Firmicutes (24\%, Lactobacillus, Streptococcus, Staphylococcus), Proteobacteria (17\%, Paracoccus, Hematobacter) and Bacteroidetes (7\%, Prevotella,
Porphyromonas). As expected, each microorganism was isolated from niches where local conditions (moisture level, $\mathrm{pH}$, lipid profile etc.) favoured its growth. Regardless of these differences, it must be pointed out that the healthy skin harbours extremely diverse microflora.

Similarly to gut microbiome, it has been reported that skin commensal bacteria influence many important processes, including the maturation of the immune system [3]. The analysis of prevalence of atopic diseases in children, who were raised in different environments (rural vs. municipal) led to the elaboration of the "hygiene hypothesis" $[6,7]$, according to which insufficient exposure to antigens of diverse microorganisms may lead to excessive Th2-type immune response. Cytokines produced by Th2 lymphocytes (interleukin (IL)-4, -5, -13) are strategic players in the vicious cycle of atopic diseases. They are also known to aggravate AD through many mechanisms [8-10].

Non-altered microbiome helps in maintaining skin homeostasis. Naik et al. have proven that skin commensals provide a specific "training" for the immunological system [11]. Their presence results in increased signalling through IL-1R which inhibits Th2 polarization and ensures correct immunological response to pathogenic microorganisms. Furthermore, specific bacteria are capable of inhibiting the growth of potentially harmful microorganisms e.g. by producing bacteriocins and preventing biofilm formation. These mechanisms will be presented below in more detail.

Address for correspondence: Leszek Blicharz MD, Department of Dermatology, Medical University of Warsaw, 82 A Koszykowa St, 05-077 Warsaw, Poland, phone: +48 5177996 23, e-mail: Iblicharz@gmail.com

Received: 15.05.2018, accepted: 11.07.2018. 


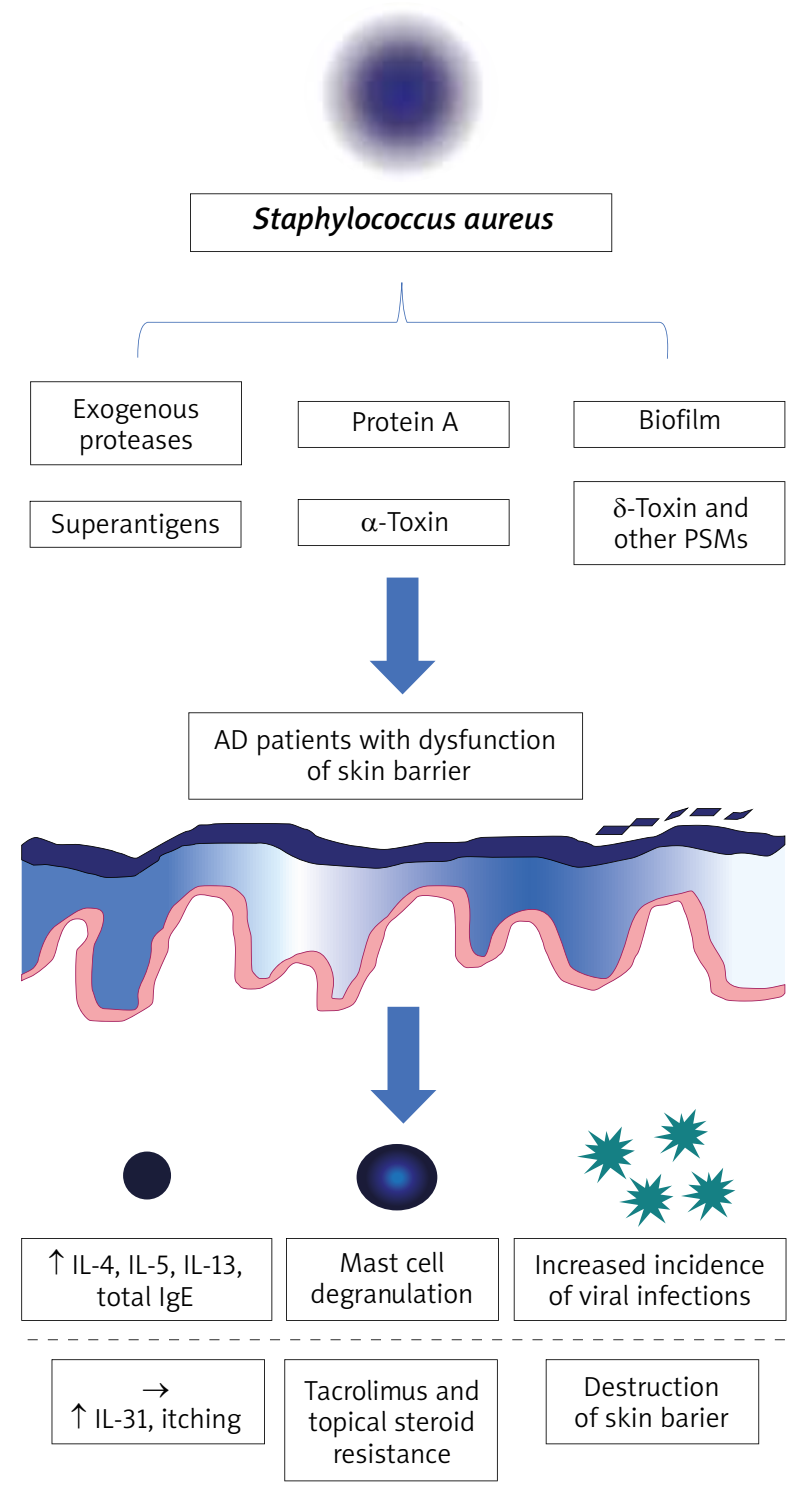

Figure 1. Virulence factors of Staphylococcus aureus and their role in the pathogenesis of AD. Colonization by S. aureus is facilitated in patients with $A D$ due to dysfunction of the epithelial barrier. Virulence factors of $S$. aureus contribute to the vicious cycle of $A D$ (see the text for reference)

\section{Genetic disorders hampering the control of bacterial pathogens}

Pattern recognition receptors (PRR) are a conservative element of the innate immune system. They bind pathogen-associated molecular patterns (PAMP) and danger-associated molecular patterns (DAMP) [12]. PRR-

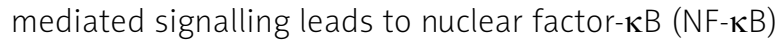
activation which results in production of pro-inflammatory cytokines and anti-microbial peptides (AMP). Toll-like receptors (TLR) are a PRR subtype, which orchestrate an appropriate immune response. Some reports have shown down-regulation of TLR2 (S. aureus-antigen binding re- ceptor) in keratinocytes and monocytes of patients with AD $[13,14]$. Reduced TLR2 expression has been associated with decreased production of IL-1B, tumor necrosis factor- $\alpha$ (TNF- $\alpha$ ) and IL-8 by monocytes [14]. Additionally, TLR2 seems to be important for maintaining the skin barrier function as $S$. aureus antigens and synthetic TLR2 agonists have been shown to stimulate the production of tight-junction proteins (claudin-1 and claudin-23) [15]. Of note, patients with short-term $S$. aureus colonization tend to have an increased expression of claudin-1 and -4, while prolonged presence of this pathogen on the skin down-regulates their expression and negatively affects the barrier function provided by tight-junctions [16]. Clinical importance of these observations remains unclear.

Destruction of the epithelial barrier and increased skin permeability are another prominent characteristics of AD which predispose to bacterial colonization. The patients with atopic dermatitis show deficiencies in two crucial components of the skin barrier: ceramides and intracellular connections [17-20]. While they are not directly related to negative microbiome fluctuations, they facilitate them for numerous reasons:

1) Epithelium damage leads to the increased expression of adhesive molecules [21-23]. This, on the other hand, favours adherence of pathogenic bacteria to the skin.

2) Increased skin permeability enables local penetration of allergens and irritants. They induce in-situ inflammation which further debilitates immune responses and epithelial regeneration $[24,25]$.

3) Trans-epidermal water loss (TWLE) and skin dryness are increased, which combined with local inflammation causes pruritus and the secondary barrier damage due to scratching [26].

Recent genetic analyses performed by Japanese and German scientists have proven the existence of numerous polymorphisms predisposing to $A D$, some of which are related to the immune system functioning $[27,28]$. Nevertheless, some data suggest that investigation should not be limited to coding sequences of the human genome only. Gene expression may also be deficient because of epigenetic disorders or interference of miRNA in the process of translation. miRNAs have been reported to regulate the expression of TLRs and influence mechanisms of responses to superantigens. These observations may justify the attempts of targeting them to reduce AD symptoms [29-31].

\section{Microbiome characteristics in atopic dermatitis}

Many diseases alter natural composition of the microbiome. Two well-known examples include selective expansion of Clostridium difficile in antibiotic-associated diarrhoea [32] or excessive development of diverse anaerobic microflora with simultaneous reduction in Lactobacilli in bacterial vaginosis [33]. Atopic dermatitis is characterized by specific changes of the microbiome as well. 
Classic culture methods have revealed that skin lesions in $\mathrm{AD}$ are colonized by $\mathrm{S}$. aureus in up to $90 \%$ of patients and that this effect is the most prominent in samples collected during disease exacerbation [34, 35]. These observations have been confirmed by the analysis of bacterial 165 rRNA [36]. Staphylococcus aureus is associated with the reduction in total microbiome diversity which positively correlates with disease severity. Therapeutic interventions that restore natural skin microflora are known to alleviate the symptoms of $A D[37,38]$. Recent investigations in children imply that colonization of the skin by S. aureus may not only be an exacerbating factor of $A D$, but also lead to its development [39]. Nonetheless, infants whose gut was colonized by S. aureus strains carrying a specific combination of superantigen and adhesin genes showed a reduced risk of subsequent development of atopic eczema, which suggests that the presence of this pathogen may globally promote appropriate maturation of the immune system [40].

Much effort has also been dedicated to determining the role of $S$. epidermidis in AD. Unlike S. aureus, colonization by commensal staphylococci (e.g. S. epidermidis) at the age of 2 months was associated with a decreased risk of AD development at the age of one year [41]. Although S. aureus expansion tends to inhibit the growth of most skin commensals, S. epidermidis remains relatively widespread in AD lesions [42]. Hon et al. have shown that aggravation of $A D$ symptoms and increased colonization of the lesional skin by S. epidermidis are associated [43]. Nevertheless, they have also reported that S. epidermidis inhibits $S$. aureus growth in the lesions. This phenomenon could be explained by the ability of S. epidermidis to stimulate AMP production through TLR2 signalling [44] and by secretion of phenol-soluble modulins (PSM) and bacteriocins that act synergically with the aforementioned in controlling pathogens such as S. aureus and S. pyogenes [45, 46]. Furthermore, S. epidermidis can inhibit formation of biofilm by $S$. aureus, while lipoteichoic acid of this specie shows immunomodulatory properties in healing wounds [47-50]. The latter stresses the importance of natural commensals in proper skin recovery and should draw the attention of future research in the context of AD lesions.

\section{Staphylococcus aureus - virulence factors affecting the disease course in atopic dermatitis}

Staphylococcus aureus not only causes skin infections, but also influences the course of AD by seemingly asymptomatic colonization. This effect can be explained by its ability to produce virulence factors such as biofilm, superantigens, $\alpha$-toxin and protein $A$ that are considered as important elements of the vicious cycle of AD.

\section{Biofilm}

Some strains of $S$. aureus are capable of biofilm formation. While this process is commonly associated with decreased effectiveness of antibiotic treatment, it may also have other consequences. Biofilm impedes clearance of pathogenic bacteria by shielding them from host immune cells such as neutrophils and macrophages. It is also known to facilitate macrophage cytotoxicity [51]. Furthermore, apoptosis has been observed after exposure of keratinocytes to staphylococcal biofilm in vitro, and substances released upon this process (TSLP, IL-4 and IL-13) showed a negative influence on the clearance of pathogenic bacteria and skin regeneration [51]. Chronic skin damage associated with $S$. aureus colonization creates a favourable environment for biofilm development [52].

\section{Exogenous proteases}

Epithelial barrier integrity may be impaired by exogenous proteases of $S$. aureus which eases the penetration of environmental antigens into deeper compartments of the skin $[53,54]$. It makes the lesions of patients with $A D$ prone to exacerbation in the presence of allergic substances and other irritants. Additionally, recent studies have focused on the ability of $S$. aureus to penetrate into sub-epidermal skin layers. Nakatsuji et al. have found that this process depends on the viability of $S$. aureus strains and their ability to produce proteases [55]. Presence of $S$. aureus in the dermis has been correlated with an increased expression of IL-4, IL-13, IL-22 and TSLP as well as with a decreased production of cathelicidin [55]. Another study suggests that SspA/V8 protease is the main substance compromising the epithelial barrier [56]. This process is prevented by IL-1B production and subsequent secretion of human b-defensin 2 (hBD2), giving light to elaborate novel therapies. Moreover, Sonesson et al. have discovered that $S$. aureus can produce another protease group, staphopains, which inactivate AMPs (LL-37) responsible for the degradation of bacterial biofilm [52].

\section{Staphylococcal superantigens}

Some strains of S. aureus produce superantigens. The most important ones include staphylococcal enterotoxin $A$ and $B(S E A / B)$ and the toxic shock syndrome toxin-1 (TSST-1) that are known to affect the course of AD in a negative way [57-65]. These molecules are bound by local antigen presenting cells (APCS) and presented to other elements of the immune system via major histocompatibility complex class 2 (MHC-II) [64]. They cause a chaotic inflammatory response by activating a large percentage of naive $T$ lymphocytes, which leads to excessive Th2 cytokine release $[57,60]$. It has also been demonstrated that despite their regulatory phenotype, CD4+ FOXP3 cells present in the skin of patients with AD can be stimulated to secrete Th2 cytokines after exposure to staphylococcal superantigens [66]. Furthermore, SEB (but also other staphylococcal antigens, such as lipoteichoic acid) has been associated with increased synthesis of IgE $[64,65,67]$. This supports the theory that the presence 
of S. aureus on the skin can provoke AD flares instead of being just an exacerbating factor. In the study conducted by Hon et al. [68], SEB-specific IgE concentration has been positively correlated with disease severity. Another negative function of SEB includes its availability to induce monocyte apoptosis by up-regulating TNF- $\alpha$ [69]. It has also been reported that $S$. aureus superantigens can contribute to the effect of glucocorticoid and tacrolimus resistance, which is especially troubling from the therapeutic point of view $[62,63]$.

Selected staphylococcal superantigens are also involved in the pathogenesis of itch in AD. IL-31, a molecule whose levels have been correlated with the intensity of pruritus, has been shown to be up-regulated after exposure to staphylococcal superantigens in vivo and more particularly to SEB (in vitro) [70]. Studies have shown that increased levels of IL-31 negatively affect the production of AMPs, which impedes S. aureus clearance [71]. What is more, skin damage and keratinocyte necrosis caused by constant scratching can additionally lead to sensitization to autoantigens, which drives the vicious cycle of AD.

\section{$\alpha-$ Toxin}

Staphylococcal $\alpha$-toxin (also known as $\alpha$-hemolysin) is highly cytotoxic to keratinocytes by leading to intrinsic caspase activation and cytochrome $c$ release from the mitochondria in experimental studies [72]. It has been demonstrated that keratinocytes exposed to Th2 cytokines are more susceptible to apoptosis through this mechanism [73]. Hong et al. have shown that $\alpha$-toxin induces atopic dermatitis-like skin inflammation and disruption of the skin barrier [74]. $\alpha$-Toxin production by S. aureus positively correlates with disease severity and has been proposed as a potential diagnostic and therapeutic target for the control of AD. Moreover, $\alpha$-hemolysin is upregulated upon formation of biofilm following neutrophil exposure and causes neutrophil inhibition. Interestingly, it also facilitates viral entry into the cells. It may be one of the reasons why patients with $A D$ are predisposed to infections with pathogens such as Molluscum contagiosum or Herpesviridae (increased incidence of eczema herpeticum) $[42,72,75]$.

\section{$\delta$-Toxin and other phenol-soluble modulins (PSMs)}

Phenol-soluble modulins (PSMs) are a family of short peptides produced by staphylococci [76]. PSMs secreted by $S$. aureus show cytolytic activity in leukocytes and are thought to play an important role in the pathogenesis of AD. $\delta$-Toxin of $S$. aureus is one of the PSMs investigated in this context. The study conducted by Nakamura et al. [77] has revealed that exposure of mast cells to $\delta$-toxin leads to their degranulation rather than lysis. Excessive IL-4 and IgE production with simultaneous exacerbation of Th2-mediated inflammation in the skin has been observed as well [77]. The PSMs have also been shown to cause keratinocyte lysis with subsequent release of proinflammatory cytokines such as IL-18 and IL-1 [78].

\section{Staphylococcal protein A}

Staphylococcal protein A is constitutively expressed on the surface of $S$. aureus and contributes to evasion of host immune responses [79]. Jun et al. have reported that it is highly expressed in membrane vesicles of $S$. aureus, whose application to AD-like skin lesions induces eczematous dermatitis. Based on this observation, the authors suggested that $S$. aureus may be an inducing factor in AD. Another study has revealed that protein A along with toxins of $S$. aureus induce local inflammation mediated by TNF- $\alpha[80]$.

\section{Novel therapeutic methods with the aim of restoring natural composition of the skin microbiome}

Increasing knowledge about the significance of disordered skin microbiome in AD is prompting the search for new treatment methods. Routine management of patients with AD with antibiotics would be contraindicated mainly because of the extent and chronic course of disease, cost-effectiveness, selection of drug-resistant strains of pathogenic bacteria [81, 82] and reduction of commensal microflora. In recent years many groundbreaking strategies have been developed. For example, lysate of a recently discovered Gram-thermal spring bacterium called Vitreoscilla filiformis has been reported to significantly reduce the symptoms of $A D$ and seborrheic dermatitis [83-85]. Volz et al. [86] have revealed that $\checkmark$. filiformis antigens induce IL10+ dendritic cells. They are a source of stimuli responsible for naïve CD4+ lymphocytes maturation into Treg1 lymphocytes. Treg1 lymphocytes produce IL-10, a cytokine known for its strongly immunosuppressive properties. This process depends on TLR2 signalling, which has already been mentioned in the context of AD-specific disorders of the innate immune system [87]. Another trial has revealed that application of lotion with heat-treated probiotic strain Lactobacillus johnsonii for 3 weeks controlled S. aureus colonization and reduced disease severity measured with the SCORAD index [88]. Interestingly, a higher concentration of $S$. aureus at baseline was associated with good response to the treatment. Nakatsuji et al. [89] have analysed the relation between the presence of commensal coagulase-negative staphylococci (CoNS) S. epidermidis and S. hominis and skin colonization by S. aureus. They have found that CoNS produce lantibiotics, i.e. prokaryotic antimicrobials that along with human AMPs (LL-37) lead to the clearance of $S$. aureus in animal and human models [89]. Favourable results have also 
been reported for topical microbiome transplantation with Roseomonas mucosa [90].

Other trials have focused on a potential therapeutic effect of synthetic AMPs. In the study conducted by Dawgul et al. [91], citropin 1.1 and temporin A appeared to be active against all S. aureus strains. Furthermore, unlike conventional antimicrobials, they did not induce resistance and showed anti-biofilm activity. The study of several AMPs introduced in catheter infections caused by biofilm-forming S. aureus has revealed high effectiveness of the treatment, especially in case of D-Bac8c2,5Leu [92]. Additionally, some promising data have come from the research over all-D omiganan, which has shown bactericidal activity towards S. aureus and satisfactory half-life in vivo [93].

Another promising direction of research includes phagolysins, i.e. anti-infectives derived from bacteriophages which have been shown to resensitize bacteria to antibiotics [94, 95]. Totté et al. [96] published a case series reporting successful treatment of MRSA infections with Endolysin Staphefekt SA.100. Results of a multi-centre randomized trial designed to evaluate the influence of Staphefekt on the use of corticosteroids, disease severity, quality of life and composition of the microbiome in patients with $A D$ are to be expected shortly [97]. Finally, Baldry et al. have managed to block production of $\alpha$-toxin by inhibiting agr signalling and have subsequently obtained reduction of inflammation in an animal model [98]. Nevertheless, an additional studies to verify the clinical application of these novel therapies in $A D$ is needed.

\section{Conclusions}

Despite its complexity, pathogenesis of atopic dermatitis is quite obviously associated with the skin microbiome. The role of $S$. aureus in the vicious cycle of AD seems to be exceptionally pronounced. S. aureus eliminates commensal bacteria from the skin, while its virulence factors show a negative effect on the epithelial barrier integrity and immune system functioning. It is possible that $S$. aureus is not only a secondary exacerbating factor but it is also one of the reasons why AD flares occur. Physiologically, the microbiome is responsible for the maintenance of immunological homeostasis and reduction of skin colonization by pathogenic bacteria. Therefore, therapies aimed to restore natural composition of the skin microbiome are being elaborated.

\section{Conflict of interest}

The authors declare no conflict of interest.

\section{References}

1. Leung DY. New insights into atopic dermatitis: role of skin barrier and immune dysregulation. Allergol Int 2013; 62: 151-61.

2. Werfel T, Allam JP, Biedermann T, et al. Cellular and molecular immunologic mechanisms in patients with atopic dermatitis. J Allergy Clin Immunol 2016; 138: 336-49.
3. Grice EA, Segre JA. The skin microbiome. Nat Rev Microbiol 2011; 9: 244-53.

4. Adamczyk K, Garncarczyk A, Antończak P. The microbiome of the skin. Dermatol Rev 2018; 105: 285-97.

5. Grice EA, Kong HH, Conlan S, et al. Topographical and temporal diversity of the human skin microbiome. Science 2009; 324: 1190-2.

6. Bach JF. The effect of infections on susceptibility to autoimmune and allergic diseases. N Engl I Med 2002; 347: 911-20.

7. Braun-Fahrländer C, Riedler J, Herz U, et al. Environmental exposure to endotoxin and its relation to asthma in school-age children. Allergy 2007; 62: 1387-93.

8. Rivers DA, Stern R, Maibach HI. A defective inflammatory response may underlie cases of atopic dermatitis. J Eur Acad Dermatol Venereol 2016 Dec 21 [Epub Ahead of print].

9. De Vuyst É, Mound A, Lambert de Rouvroit C, Poumay Y. Modelling atopic dermatitis during the morphogenetic process involved in reconstruction of a human epidermis. Curr Res Transl Med 2016; 64: 179-83.

10. Hönzke S, Wallmeyer L, Ostrowski A, et al. Influence of Th2 cytokines on the cornified envelope, tight junction proteins, and beta-defensins in filaggrin-deficient skin equivalents. J Invest Dermatol 2016; 136: 631-9.

11. Naik S, Bouladoux N, Wilhelm C, et al. Compartmentalized control of skin immunity by resident commensals. Science 2012; 337: 1115-9.

12. Kuo IH, Yoshida T, De Benedetto A, Beck LA. The cutaneous innate immune response in patients with atopic dermatitis. J Allergy Clin Immunol 2013; 131: 266-78.

13. Hasannejad H, Takahashi R, Kimishima M, et al. Selective impairment of Toll-like receptor 2-mediated proinflammatory cytokine production by monocytes from patients with atopic dermatitis. J Allergy Clin Immunol 2007; 120: 69-75.

14. Niebuhr M, Lutat C, Sigel S, Werfel T. Impaired TLR-2 expression and TLR-2-mediated cytokine secretion in macrophages from patients with atopic dermatitis. Allergy 2009; 64: 1580-7.

15. Kuo IH, Carpenter-Mendini A, Yoshida T, et al. Activation of epidermal toll-like receptor 2 enhances tight junction function: implications for atopic dermatitis and skin barrier repair. I Invest Dermatol 2013; 133: 988-98.

16. Bäsler K, Galliano MF, Bergmann S, et al. Biphasic influence of Staphylococcus aureus on human epidermal tight junctions. Ann N Y Acad Sci 2017; 1405: 53-70.

17. Ishikawa J, Narita H, Kondo N, et al. Changes in the ceramide profile of atopic dermatitis patients. I Invest Dermatol 2010; 130: 2511-4.

18. van Smeden J, Janssens M, Kaye EC, et al. The importance of free fatty acid chain length for the skin barrier function in atopic eczema patients. Exp Dermatol 2014; 23: 45-52.

19. Bäsler K, Brandner JM. Tight junctions in skin inflammation. Pflugers Arch 2017; 469: 3-14.

20. Gruber R, Börnchen C, Rose K, et al. Diverse regulation of claudin-1 and claudin-4 in atopic dermatitis. Am J Pathol 2015; 185: 2777-89.

21. Sinha B, François PP, Nüsse O, et al. Fibronectin-binding protein acts as Staphylococcus aureus invasin via fibronectin bridging to integrin alpha5beta1. Cell Microbiol 1999; 1: 101-17.

22. Cho SH, Strickland I, Boguniewicz M, Leung DY. Fibronectin and fibrinogen contribute to the enhanced binding of Staphylococcus aureus to atopic skin. J Allergy Clin Immunol 2001; 108: 269-74.

23. Fleury OM, McAleer MA, Feuillie C, et al. Clumping factor B promotes adherence of Staphylococcus aureus to corneocytes in atopic dermatitis. Infect Immun 2017; 85: pii: e00994-16. 
24. Ogawa H, Yoshiike T. A speculative view of atopic dermatitis: barrier dysfunction in pathogenesis. J Dermatol Sci 1993; 5: 197-204.

25. Berard F, Marty JP, Nicolas JF. Allergen penetration through the skin. Eur J Dermatol 2003; 13: 324-30.

26. Kamata Y, Tominaga M, Takamori K. Itch in atopic dermatitis management. Curr Probl Dermatol 2016; 50: 86-93.

27. Hirota T, Takahashi A, Kubo M, et al. Genome-wide association study identifies eight new susceptibility loci for atopic dermatitis in the Japanese population. Nat Genet 2012; 44: 1222-6.

28. Ellinghaus D, Baurecht H, Esparza-Gordillo J, et al. High-density genotyping study identifies four new susceptibility loci for atopic dermatitis. Nat Genet 2013; 45: 808-12.

29. Virtue A, Wang H, Yang XF. MicroRNAs and toll-like receptor/interleukin-1 receptor signaling. J Hematol Oncol 2012; 5: 66.

30. He X, Jing Z, Cheng G. MicroRNAs: new regulators of Toll-like receptor signalling pathways. Biomed Res Int 2014; 2014: 945169

31. O'Neill LA, Sheedy FJ, McCoy CE. MicroRNAs: the fine-tuners of Toll-like receptor signalling. Nat Rev Immunol 2011; 11: 163-75.

32. Malnick SD, Zimhony O. Treatment of Clostridium difficile-associated diarrhea. Ann Pharmacother 2002; 36: 1767-75.

33. Fredricks DN, Fiedler TL, Marrazzo JM. Molecular identification of bacteria associated with bacterial vaginosis. N Engl J Med 2005; 353: 1899-911.

34. Leyden JJ, Marples RR, Kligman AM. Staphylococcus aureus in the lesions of atopic dermatitis. Br J Dermatol 1974; 90: 525-30.

35. Park HY, Kim CR, Huh IS, et al. Staphylococcus aureus colonization in acute and chronic skin lesions of patients with atopic dermatitis. Ann Dermatol 2013; 25: 410-6.

36. Kong HH, Oh J, Deming C, et al. Temporal shifts in the skin microbiome associated with disease flares and treatment in children with atopic dermatitis. Genome Res 2012; 22: 850-9.

37. Salava A, Lauerma A. Role of the skin microbiome in atopic dermatitis. Clin Transl Allergy 2014; 4: 33.

38. Wong SM, Ng TG, Baba R. Efficacy and safety of sodium hypochlorite (bleach) baths in patients with moderate to severe atopic dermatitis in Malaysia. J Dermatol 2013; 40: 874-80.

39. Meylan P, Lang C, Mermoud S, et al. Skin colonization by Staphylococcus aureus precedes the clinical diagnosis of atopic dermatitis in infancy. J Invest Dermatol 2017; 137: 2497-504.

40. Nowrouzian FL, Lina G, Hodille E, et al. Superantigens and ad hesins of infant gut commensal Staphylococcus aureus strains and association with subsequent development of atopic eczema. Br J Dermatol 2017; 176: 439-45.

41. Kennedy EA, Connolly J, Hourihane JO, et al. Skin microbiome before development of atopic dermatitis: early colonization with commensal staphylococci at 2 months is associated with a lower risk of atopic dermatitis at 1 year. J Allergy Clin Immunol 2017; 139: 166-72.

42. Ong PY, Leung DY. Bacterial and viral infections in atopic dermatitis: a comprehensive review. Clin Rev Allergy Immunol 2016; 51: 329-37

43. Hon KL, Tsang YC, Pong NH. Exploring Staphylococcus epidermidis in atopic eczema: friend or foe? Clin Exp Dermatol 2016; 41: 659-63.

44. Lai Y, Cogen AL, Radek KA, et al. Activation of TLR2 by a small molecule produced by Staphylococcus epidermidis increases antimicrobial defense against bacterial skin infections. J Invest Dermatol 2010; 130: 2211-21.

45. Cogen AL, Yamasaki K, Sanchez KM, et al. Selective antimicrobial action is provided by phenol-soluble modulins derived from Staphylococcus epidermidis, a normal resident of the skin. J Invest Dermatol 2010; 130: 192-200.

46. Otto M. Staphylococcus colonization of the skin and antimicrobial peptides. Expert Rev Dermatol 2010; 5: 183-95.
47. Sugimoto S, Iwamoto T, Takada K, et al. Staphylococcus epidermidis Esp degrades specific proteins associated with Staphylococcus aureus biofilm formation and host-pathogen interaction. J Bacteriol 2013; 195: 1645-55.

48. Iwase T, Uehara Y, Shinji H, et al. Staphylococcus epidermidis Esp inhibits Staphylococcus aureus biofilm formation and nasal colonization. Nature 2010; 465: 346-9.

49. Lai Y, Di Nardo A, Nakatsuji T, et al. Commensal bacteria regulate Toll-like receptor 3-dependent inflammation after skin injury. Nat Med 2009; 15: 1377-82.

50. Vandecandelaere I, Depuydt P, Nelis HJ, Coenye T. Protease production by Staphylococcus epidermidis and its effect on Staphylococcus aureus biofilms. Pathog Dis 2014; 70: 321-31.

51. Gonzalez T, Biagini Myers JM, Herr AB, Khurana Hershey GK. Staphylococcal biofilms in atopic dermatitis. Curr Allergy Asthma Rep 2017; 17: 81.

52. Sonesson A, Przybyszewska K, Eriksson S, et al. Identification of bacterial biofilm and the Staphylococcus aureus derived protease, staphopain, on the skin surface of patients with atopic dermatitis. Sci Rep 2017; 7: 8689.

53. Miedzobrodzki J, Kaszycki P, Bialecka A, Kasprowicz A. ProteoIytic activity of Staphylococcus aureus strains isolated from the colonized skin of patients with acute-phase atopic dermatitis. Eur J Clin Microbiol Infect Dis 2002; 21: 269-76.

54. Takai T, Ikeda S. Barrier dysfunction caused by environmental proteases in the pathogenesis of allergic diseases. Allergol Int 2011; 60: 25-35.

55. Nakatsuji T, Chen TH, Two AM, et al. Staphylococcus aureus exploits epidermal barrier defects in atopic dermatitis to trigger cytokine expression. J Invest Dermatol 2016; 136: 2192-200.

56. Wang B, McHugh BJ, Qureshi A, et al. IL-1B induced protection of keratinocytes against Staphylococcus aureus-secreted proteases is mediated by human b-defensin 2. J Invest Dermatol 2017; 137: 95-105.

57. Nada HA, Gomaa NI, Elakhras A, et al. Skin colonization by superantigen-producing Staphylococcus aureus in Egyptian patients with atopic dermatitis and its relation to disease severity and serum interleukin-4 level. Int J Infect Dis 2012; 16: e29-33.

58. Na SY, Roh JY, Kim JM, et al. Analysis of colonization and genotyping of the exotoxins of Staphylococcus aureus in patients with atopic dermatitis. Ann Dermatol 2012; 24: 413-9.

59. Xu SX, McCormick JK. Staphylococcal superantigens in colonization and disease. Front Cell Infect Microbiol 2012; 2: 52.

60. Lehmann HS, Heaton T, Mallon D, Holt PG. Staphylococcal enterotoxin-B-mediated stimulation of interleukin-13 production as a potential aetiologic factor in eczema in infants. Int Arch Allergy Immunol 2004; 135: 306-12.

61. Gould HJ, Takhar P, Harries HE, et al. The allergic march from Staphylococcus aureus superantigens to immunoglobulin E. Chem Immunol Allergy 2007; 93: 106-36.

62. Schlievert PM, Case LC, Strandberg KL, et al. Superantigen profile of Staphylococcus aureus isolates from patients with steroid-resistant atopic dermatitis. Clin Infect Dis 2008; 46: 1562-7.

63. Fukushima H, Hirano T, Shibayama N, et al. The role of immune response to Staphylococcus aureus superantigens and disease severity in relation to the sensitivity to tacrolimus in atopic dermatitis. Int Arch Allergy Immunol 2006; 141: 281-9.

64. Krogman A, Tilahun A, David CS, et al. HLA-DR polymorphisms influence in vivo responses to staphylococcal toxic shock syndrome toxin-1 in a transgenic mouse model. HLA 2017; 89: 20-8.

65. Orfali RL, Sato MN, Santos VG, et al. Staphylococcal enterotoxin B induces specific IgG4 and IgE antibody serum levels in atopic dermatitis. Int J Dermatol 2015; 54: 898-904.

66. Lin YT, Wang CT, Chao PS, et al. Skin-homing CD4+ Foxp3+ T cells exert Th2-like function after staphylococcal superantigen 
stimulation in atopic dermatitis patients. Clin Exp Allergy 2011; 41: $516-25$

67. Matsui K, Nishikawa A. Lipoteichoic acid from Staphylococcus aureus enhances allergen-specific immunoglobulin E production in mice. Clin Exp Allergy 2003; 33: 842-8.

68. Hon KL, Tsang KY, Kung JS, et al. Clinical signs, staphylococcus and atopic eczema-related seromarkers. Molecules 2017; 22: pii: E291.

69. Zhang X, Shang W, Yuan J, et al. Positive feedback cycle of TNFalpha promotes staphylococcal enterotoxin B-induced THP-1 cell apoptosis. Front Cell Infect Microbiol 2016; 6: 109.

70. Sonkoly E, Muller A, Lauerma Al, et al. IL-31: a new link between T cells and pruritus in atopic skin inflammation. J Allergy Clin Immunol 2006; 117: 411-7.

71. van Drongelen V, Haisma EM, Out-Luiting JJ, et al. Reduced filaggrin expression is accompanied by increased Staphylococcus aureus colonization of epidermal skin models. Clin Exp Allergy 2014; 44: 1515-24.

72. Bin L, Kim BE, Brauweiler A, et al. Staphylococcus aureus alphatoxin modulates skin host response to viral infection. J Allergy Clin Immunol 2012; 130: 683-91.e2.

73. Brauweiler AM, Goleva E, Leung DYM. Th2 cytokines increase Staphylococcus aureus alpha toxin-induced keratinocyte death through the signal transducer and activator of transcription 6 (STAT6). J Invest Dermatol 2014; 134: 2114-21.

74. Hong SW, Choi EB, Min TK, et al. An important role of alphahemolysin in extracellular vesicles on the development of atopic dermatitis induced by Staphylococcus aureus. PLoS One 2014; 9: e100499.

75. Olsen JR, Piguet V, Gallacher J, Francis NA. Molluscum contagiosum and associations with atopic eczema in children: a retrospective longitudinal study in primary care. Br J Gen Pract 2016; 66: e53-8.

76. Cheung GY, Joo HS, Chatterjee SS, Otto M. Phenol-soluble modulins - critical determinants of staphylococcal virulence. FEMS Microbiol Rev 2014; 38: 698-719.

77. Nakamura Y, Oscherwitz J, Cease KB, et al. Staphylococcus deltatoxin induces allergic skin disease by activating mast cells. $\mathrm{Na}$ ture 2013; 503: 397-401

78. Syed AK, Reed TJ, Clark KL, et al. Staphlyococcus aureus phenolsoluble modulins stimulate the release of proinflammatory cytokines from keratinocytes and are required for induction of skin inflammation. Infect Immun 2015; 83: 3428-37.

79. Votintseva AA, Fung R, Miller RR, et al. Prevalence of Staphylococcus aureus protein A (spa) mutants in the community and hospitals in Oxfordshire. BMC Microbiol 2014; 14: 63.

80. Ezepchuk YV, Leung DY, Middleton MH, et al. Staphylococcal toxins and protein A differentially induce cytotoxicity and release of tumor necrosis factor-alpha from human keratinocytes. J Invest Dermatol 1996; 107: 603-9.

81. Harkins CP, McAleer MA, Bennett D, et al. The widespread use of topical antimicrobials enriches for resistance in Staphylococcus aureus isolated from atopic dermatitis patients. Br J Dermatol 2018; 179: 951-8.

82. Błażewicz I, Jaśkiewicz M, Piechowicz L, et al. Activity of antimicrobial peptides and conventional antibiotics against superantigen positive Staphylococcus aureus isolated from patients with atopic dermatitis. Adv Dermatol Allergol 2018; 35: 74-82.

83. Guéniche A, Hennino A, Goujon C, et al. Improvement of atopic dermatitis skin symptoms by Vitreoscilla filiformis bacterial extract. Eur J Dermatol 2006; 16: 380-4.

84. Guéniche A, Cathelineau AC, Bastien P, et al. Vitreoscilla filiformis biomass improves seborrheic dermatitis. J Eur Acad Dermatol Venereol 2008; 22: 1014-5.
85. Gueniche A, Knaudt B, Schuck E, et al. Effects of nonpathogenic Gram-negative bacterium Vitreoscilla filiformis lysate on atopic dermatitis: a prospective, randomized, double-blind, placebocontrolled clinical study. Br J Dermatol 2008; 159: 1357-63.

86. Volz T, Skabytska Y, Guenova E, et al. Nonpathogenic bacteria alleviating atopic dermatitis inflammation induce IL-10-producing dendritic cells and regulatory Tr1 cells. I Invest Dermatol 2014; 134: 96-104.

87. Mahe YF, Perez MJ, Tacheau C, et al. A new Vitreoscilla filiformis extract grown on spa water-enriched medium activates endogenous cutaneous antioxidant and antimicrobial defenses through a potential Toll-like receptor 2/protein kinase C, zeta transduction pathway. Clin Cosmet Investig Dermatol 2013; 6: 191-6.

88. Blanchet-Réthoré S, Bourdès V, Mercenier A, et al. Effect of a lotion containing the heat-treated probiotic strain Lactobacillus johnsonii NCC 533 on Staphylococcus aureus colonization in atopic dermatitis. Clin Cosmet Investig Dermatol 2017 10: 249-57.

89. Nakatsuji T, Chen TH, Narala S, et al. Antimicrobials from human skin commensal bacteria protect against Staphylococcus aureus and are deficient in atopic dermatitis. Sci Transl Med 2017; 9: pii: eaah4680. doi: 10.1126/scitranslmed.aah4680.

90. Myles IA, Earland NJ, Anderson ED, et al. First-in-human topical microbiome transplantation with Roseomonas mucosa for atopic dermatitis. JCl Insight 2018; 3: pii: 120608.

91. Dawgul M, Baranska-Rybak W, Piechowicz L, et al. The antistaphylococcal activity of citropin 1.1 and temporin a against planktonic cells and biofilms formed by isolates from patients with atopic dermatitis: an assessment of their potential to induce microbial resistance compared to conventional antimicrobials. Pharmaceuticals (Basel) 2016; 9: pii: E30. doi: 10.3390/ ph9020030

92. Zapotoczna M, Forde É, Hogan S, et al. Eradication of Staphylococcus aureus biofilm infections using synthetic antimicrobial peptides. J Infect Dis 2017; 215: 975-83.

93. Ng SMS, Teo SW, Yong YE, et al. Preliminary investigations into developing all-D Omiganan for treating Mupirocin-resistant MRSA skin infections. Chem Biol Drug Des 2017; 90: 1155-60.

94. Fischetti VA. Lysin therapy for staphylococcus aureus and other bacterial pathogens. Curr Top Microbiol Immunol 2017; 409: 529-40.

95. Schuch R, Lee HM, Schneider BC, et al. Combination therapy with lysin CF-301 and antibiotic is superior to antibiotic alone for treating methicillin-resistant Staphylococcus aureus-induced murine bacteremia. I Infect Dis 2014; 209: 1469-78.

96. Totté JEE, van Doorn MB, Pasmans SGMA. successful treatment of chronic Staphylococcus aureus-related dermatoses with the topical endolysin staphefekt SA.100: a report of 3 cases. Case Rep Dermatol 2017; 9: 19-25.

97. Totté J, de Wit J, Pardo L, et al. Targeted anti-staphylococcal therapy with endolysins in atopic dermatitis and the effect on steroid use, disease severity and the microbiome: study protocol for a randomized controlled trial (MAAS trial). Trials 2017; 18: 404.

98. Baldry M, Nakamura Y, Nakagawa S, et al. Application of an agrspecific anti-virulence compound as therapy for Staphylococcus aureus-induced inflammatory skin disease. J Infect Dis 2018; 218: 1009-13. 